\title{
COAL CONTRACTOR MINING FACTS - 2007
}

An independent contractor is defined by the Mine Safety and Health Administration (MSHA) as "any person, partnership, corporation, subsidiary of a corporation, firm, association or other organization that contracts to perform services or construction at a mine." Contractors that perform specific types of work are required to report the number of employees who work at coal mines and noncoal mines.

\section{Companies}

In 2007, a total of 2,999 contracting companies reported employment at coal mines to MSHA, or $35.7 \%$ of all independent contracting companies.

\section{Employees}

A total of 38,865 employees, ${ }^{1}$ corresponding to 22,741 full-time equivalent (FTE) ${ }^{2}$ employees, were reported by coal contractors to MSHA.

- Within the mining sectors, ${ }^{3}$ coal contractor employees comprised $6.8 \%$ of all employee hours reported to MSHA.

- Coal contractor employee hours were reported for both underground (13.5\%) and surface $(86.5 \%)$ work locations. ${ }^{4}$

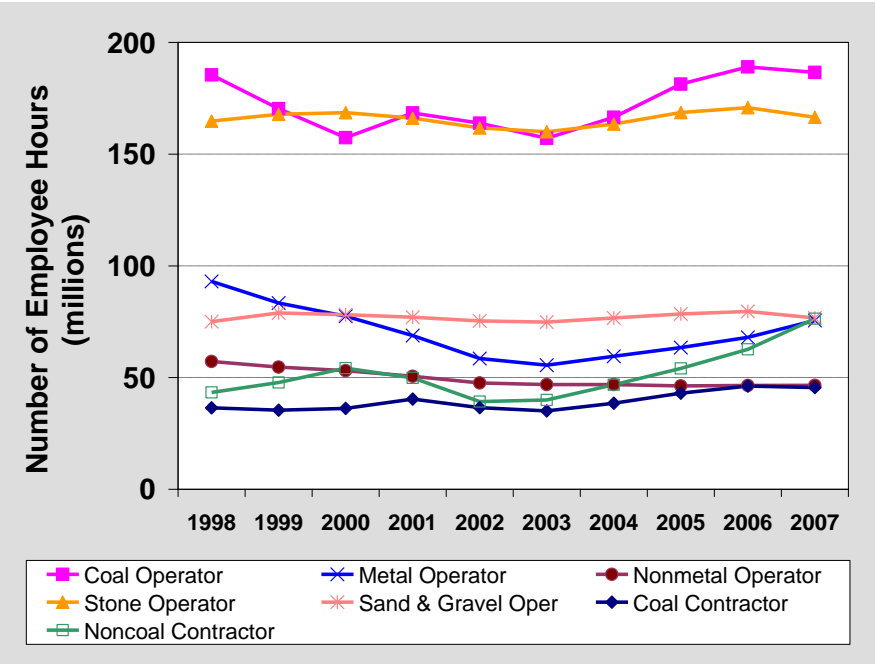

\section{Fatalities}

Six occupational fatalities occurred among coal contractor employees in 2007. These coal fatalities accounted for $33.3 \%$ of all contractor fatalities.

- The coal contractor fatality rate was 28.0 fatalities per 100,000 FTE employees. The underground rate was $97.7(n=3)$ compared to a rate of $16.3(n=3)$ for surface work locations.

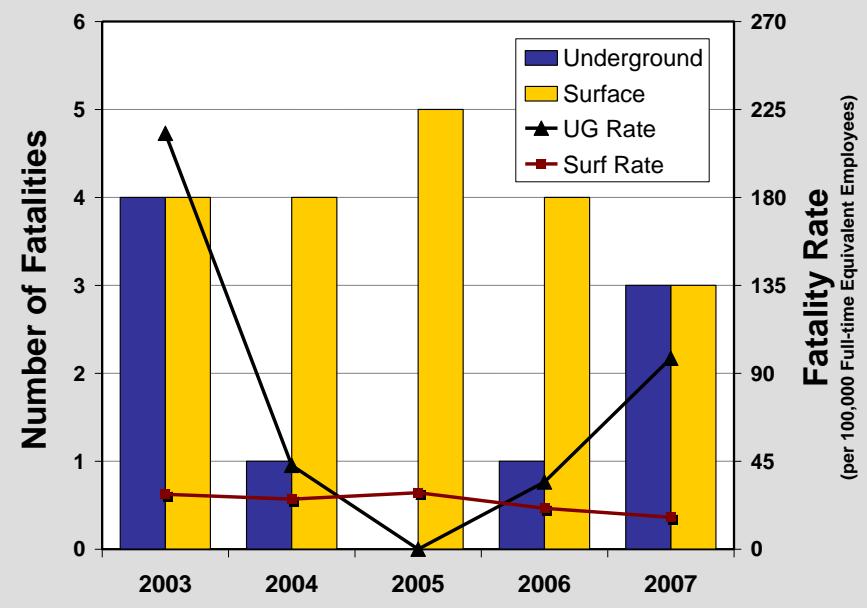

Nonfatal Lost-time Injuries

There were 460 nonfatal lost-time injuries among coal contractor employees (177 at underground and 283 at surface work locations) with an overall rate of 2.1 injuries per 100 FTE employees. These injuries resulted in 28,071 days lost from work. ${ }^{5}$

- The underground nonfatal lost-time injury rate was greater than the surface injury rate (5.8 vs. 1.5 per 100 FTE workers).

- The most frequent classification of nonfatal losttime injuries for coal contractor employees involved handling materials $(n=143 ; 31.1 \%)$, followed by slip or fall of person $(n=101 ; 22.0 \%)$.

- Sprains and strains were the most frequently reported nature of injury $(n=169 ; 36.7 \%)$.

- The back was the most frequently reported body part injured $(n=64 ; 13.9 \%)$ and accounted for 4,914 days lost from work.

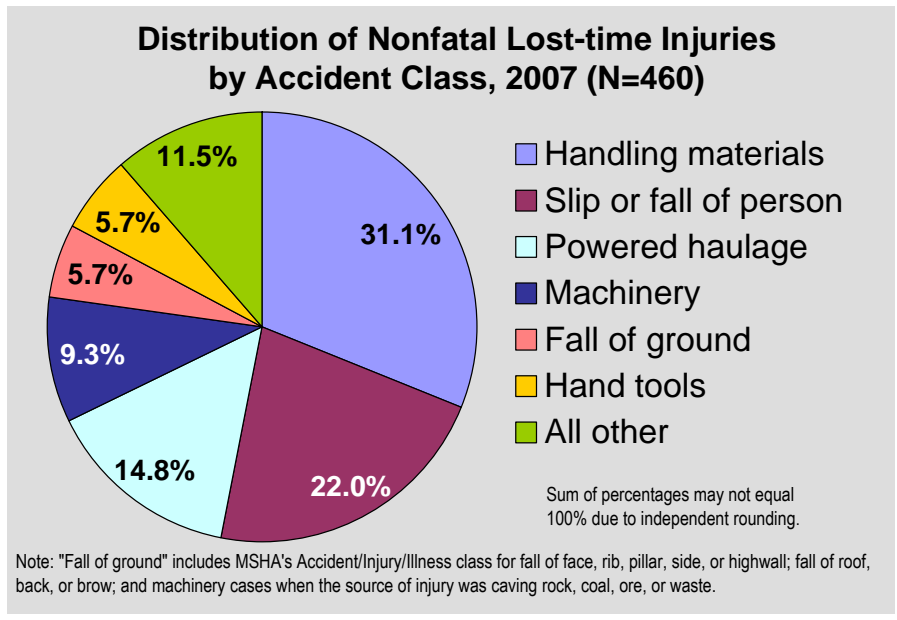




\section{Mine and Employment Characteristics, 2007}

\begin{tabular}{|c|c|c|c|c|c|c|c|c|c|}
\hline \multirow{2}{*}{$\begin{array}{l}\text { Commodity and } \\
\text { Type of Employer }\end{array}$} & \multicolumn{3}{|c|}{ Number of Mining Operations ${ }^{1}$} & \multicolumn{3}{|c|}{ Number of Employees ${ }^{2}$} & \multicolumn{3}{|c|}{ Number of FTE Employees ${ }^{3}$} \\
\hline & Underground & Surface & Total & Underground & Surface & Total & Underground & Surface & Total \\
\hline Coal Operator & 631 & 1,399 & 2,030 & 37,829 & 46,242 & 84,071 & 42,238 & 51,027 & 93,265 \\
\hline Metal Operator & 97 & 181 & 278 & 5,333 & 30,667 & 36,000 & 5,437 & 32,309 & 37,746 \\
\hline Nonmetal Operator & 48 & 677 & 725 & 2,411 & 20,453 & 22,864 & 2,478 & 20,788 & 23,265 \\
\hline Stone Operator & 113 & 4,526 & 4,639 & 1,845 & 80,873 & 82,718 & 2,063 & 81,191 & 83,254 \\
\hline Sand and Gravel Operator & NA & 7,199 & 7,199 & NA & 45,761 & 45,761 & NA & 38,340 & 38,340 \\
\hline \multirow[t]{2}{*}{ Operator Total } & 889 & 13,982 & 14,871 & 47,418 & 223,996 & 271,414 & 52,216 & 223,655 & 275,871 \\
\hline & \multicolumn{3}{|c|}{ Number of Companies } & & & & & & \\
\hline Coal Contractor & NA & NA & 2,999 & 5,160 & 33,705 & 38,865 & 3,071 & 19,670 & 22,741 \\
\hline Noncoal Contractor & NA & NA & 5,400 & 3,039 & 64,805 & 67,844 & 1,935 & 36,216 & 38,151 \\
\hline Contractor Total & NA & NA & 8,399 & 8,199 & 98,510 & 106,709 & 5,006 & 55,886 & 60,892 \\
\hline \multicolumn{4}{|l|}{ TOTAL } & 55,617 & 322,506 & 378,123 & 57,222 & 279,541 & 336,763 \\
\hline
\end{tabular}

\section{Mining Occupational Fatalities and Injuries, 2007}

\begin{tabular}{|c|c|c|c|c|c|c|c|c|c|c|c|c|}
\hline \multirow{3}{*}{$\begin{array}{l}\text { Commodity and } \\
\text { Type of Employer }\end{array}$} & \multicolumn{6}{|c|}{ Occupational Fatalities (per 100,000 FTE employees) } & \multicolumn{6}{|c|}{ Nonfatal Lost-time Injuries (per 100 FTE employees) } \\
\hline & \multicolumn{2}{|c|}{ Underground } & \multicolumn{2}{|c|}{ Surface } & \multicolumn{2}{|c|}{ Total } & \multicolumn{2}{|c|}{ Underground } & \multicolumn{2}{|c|}{ Surface } & \multicolumn{2}{|c|}{ Total } \\
\hline & No. & Rate & No. & Rate & & Rate & & Rate & No. & Rate & No. & Rate \\
\hline Coal Operator & 18 & 42.6 & 10 & 21.1 & 28 & 31.2 & $2,12 C$ & 5.0 & 822 & 1.7 & 2,942 & 3.3 \\
\hline Metal Operator & 3 & 55.2 & 4 & 14.4 & 7 & 21.1 & $17 \varepsilon$ & 3.3 & 620 & 2.2 & 798 & 2.4 \\
\hline Nonmetal Operator & 1 & 40.4 & 0 & 0.0 & 1 & 5.0 & 91 & 3.7 & 417 & 2.4 & 508 & 2.5 \\
\hline Stone Operator & 0 & 0.0 & 8 & 11.4 & 8 & 11.1 & 36 & 1.7 & 1,844 & 2.6 & 1,880 & 2.6 \\
\hline Sand and Gravel Operator & NA & NA & 5 & 15.0 & 5 & 15.0 & $\mathrm{~N} A$ & NA & 657 & 2.0 & 657 & 2.0 \\
\hline Operator Total & 22 & 42.1 & 27 & 13.8 & 49 & 19.7 & 2,425 & 4.6 & 4,360 & 2.2 & 6,785 & 2.7 \\
\hline Coal Contractor & 3 & 97.7 & 3 & 16.3 & 6 & 28.0 & 177 & 5.8 & 283 & 1.5 & 460 & 2.1 \\
\hline Noncoal Contractor & 2 & 103.4 & 10 & 29.3 & 12 & 33.3 & 43 & 2.2 & 454 & 1.3 & 497 & 1.4 \\
\hline Contractor Total & 5 & 99.9 & 13 & 24.8 & 18 & 31.3 & $22 C$ & 4.4 & 737 & 1.4 & 957 & 1.7 \\
\hline TOTAL & 27 & 47.2 & 40 & 16.1 & 67 & 21.9 & 2,645 & 4.6 & 5,097 & 2.0 & 7,742 & 2.5 \\
\hline
\end{tabular}

NA Not applicable.

Data source: Publicly released data files of employment and accident/injury/illness collected by MSHA under 30 CFR 50 .

Notes: All analyses of accident data exclude office employees. Occupational fatalities exclude all cases under 17 years of age. Further statistical methodology is available on the NIOSH Internet [http://www.cdc.gov/niosh/mining/statistics/method.htm]. Data in the above tables may not add to totals shown because of independent rounding. Caution should be used when interpreting rates based on a small number of events.

${ }^{1}$ Average number of employees working at individual mines during calendar quarters of active operations (includes office workers).

${ }^{2}$ Full-time equivalent employees computed using reported employee hours $(2,000$ hours $=1$ FTE).

${ }^{3}$ Mining sectors include coal operators, metal operators, nonmetal operators, stone operators, sand and gravel operators, coal contractors, and noncoal contractors.

${ }^{4}$ Surface work locations include surface operations at underground mines, surface operations (strip or open pit), auger, culm banks, dredge, independent shops and yards, and mills or preparation plants.

${ }^{5}$ Includes actual days away from work and/or days of restricted work activity. For permanently disabling injuries only, statutory days charged by MSHA were used if they exceeded the total lost workdays.

To receive $\mathrm{NIOSH}$ documents or more information about occupational safety and health topics, contact $\mathrm{NIOSH}$ at

1-800-CDC-INFO (1-800-232-4636)

TTY: 1-888-232-6348

e-mail: cdcinfo@cdc.gov

or visit the NIOSH Web site at http://www.cdc.gov/niosh 